\title{
A small-scale EUV jet in the quiet Sun region
}

\author{
Junchao Hong ${ }^{1,2}$, Yunchun Jiang ${ }^{1}$, Ruisheng Zheng ${ }^{1}$, and $\mathbf{Y i ~ B i}^{1}$ \\ ${ }^{1}$ National Astronomical Observatories/Yunnan Astronomical Observatory, Chinese Academy \\ of Sciences, Kunming, Yunnan 650011, P.R.China \\ ${ }^{2}$ Graduate School of Chinese Academy of Sciences, Beijing, P.R. China \\ email: hjcsolar@ynao.ac.cn
}

\begin{abstract}
Solar jets are typical proxies of small-scale magnetic reconnection events in the solar atmosphere. In this paper, we observe a small-scale jet in a solar quiet region, using data from $S D O /$ Atmospheric Imaging Assembly (AIA), Helioseismic and Magnetic Imager (HMI), with supplemental data from STEREO/EUVI. From HMI magnetograms and calculated photospheric flows, we find that the jet is related to the interaction between unipolar network fields and emerging internetwork bipoles at the boundary of a supergranular cell. In AIA extremeultraviolet images, the jet actually includes two successive plasma ejections along different directions. The first ejection follows a distorted path which guides plasma into a small filament channel nearby. However, the second one shot straight along another direction that is parallel with extrapolated potential magnetic field lines on the local. According to these observations, we advocate that during the jet eruption new emerging magnetic fields are reconnecting at the edge of the supergranular cell with different kinds of ambient fields from low (magnetic canopy) to high (high-reaching loops) to allow the occurrence of successive ejections along different directions.
\end{abstract}

Keywords. Sun: magnetic fields, Sun: evolution

\section{Introduction}

Solar jets including X-ray jets, EUV jets, $\mathrm{H} \alpha$ surges, macrospicules, are columnar eruptions in which mass is often ejected along magnetic field lines (e.g., Jiang et al. 2007, Moore et al. 2010, Hong et al. 2011, Shen et al. 2011, Shen et al. 2012, Chen et al. 2012, Yang et al. 2012). They are often trigged by small-scale magnetic reconnections between emerging bipoles and ambient unipolar flux (Shibata et al. 1992, Yokoyama \& Shibata 1996).

Over the quiet-Sun region, small-scale reconnection events such as jets occur with high frequency at the boundaries of supergranular network lanes. The supergranular network is the pattern of supergranular convection cells over the entire surface of the Sun outside of sunspots (Leighton et al. 1962). Magnetic flux in the supergranular cells (internetwork flux) are continously swept to pile up with the larger magnetic flux in cell boundaries (network flux) by convective plasma flows in the photospheric layers (Martin 1988, Wang 1988, Parnell. 2002, Innes et al. 2009). It results in the cancellation of internetwork flux with network flux occur frequently along the network lanes and thus gives rise to various transient small-scale phenomena such as spicules, microflare and jets. On the other hand, over most of the supergranular cells the magnetic field is belived to form a canopy dominated by horizontal fields (Solanki 1993) and above the magnetic canopy are the high-reaching magnetic loops throughout the solar atompsphere (Dowdy et al. 
1986). So, jets produced by reconnections of network flux with internetwork flux may be ejected along either the low magnetic canopy or the high-reaching magnetic loops.

In this paper, we analyze an extreme-ultraviolet (EUV) jet with two successive plasma ejections in the quiet Sun using on-disk observations of the Solar Dynamics Observatory (SDO) and on-limb observations of the Ahead of Solar Terrestrial Relations Observatory (STEREO-A). This event is dynamically related to emerging bipoles and supergranular flows. Its two successive ejections follow different kinds of magnetic fields rooted in the edge of the supergranular cell.

\section{Observations}

The small jet was seen from the Sun on April, 22, 2011 and tracked by both $S D O$ and STEREO-A spacecraft. The separation of the two spacecraft at this time was about $90^{\circ}$.

For detailed analysis, we adopt line-of-sight magnetograms obtained with the Helioseismic and Magnetic Imager (HMI; Schou et al. 2010) and multi-wavelength EUV images taken by the Atmospheric Imaging Assembly (AIA; Lemen et al. 2012) on board SDO. HMI magnetograms with a $45 \mathrm{~s}$ cadence, $0^{\prime \prime} .5$ pixel size and $10 \mathrm{G}$ noise were used to trace the photosphreic magnetic field evolution. The averaged flow fields of magnetic elements were calculated by using the differential affine velocity estimator (DAVE; Schuck 2006), the result of which can present supergranular flow pattern in the quiet Sun. AIA images with a pixel size of $0^{\prime \prime} .6$ and $12 \mathrm{~s}$ cadence were used to show the EUV jet on the disk. The magnetic topology extrapolated by the potential field source surface (PFSS; Schrijver \& De Rosa 2003) was also derived to correlate with the orientation of plasma ejections. Additionally, the $304 \AA$ snapshots taken by the Extreme Ultraviolet Imager (EUVI; Wuelser et al.2004) on board STEREO-A were used to show the limb counterpart of the jet, for which the spatial and temporal resolutions are $1^{\prime \prime} .6$ pixel $^{-1}$ and $10 \mathrm{~min}$ cadence, respectively.

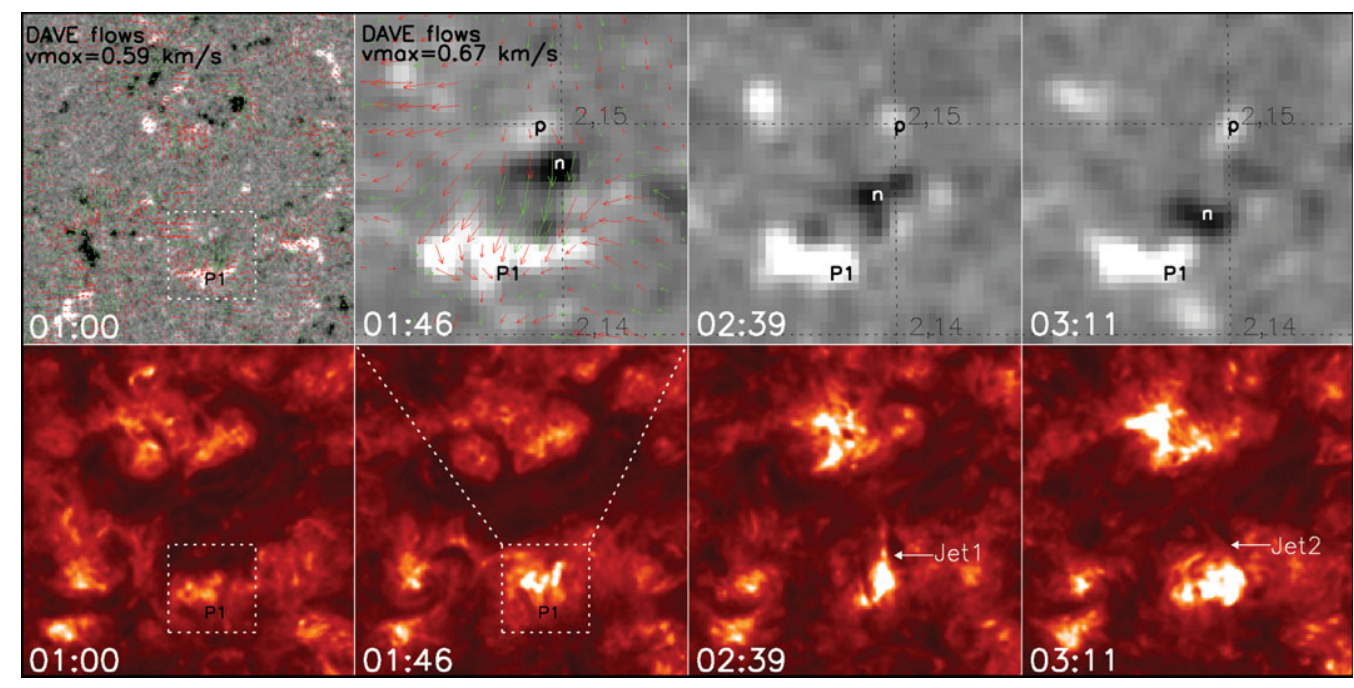

Figure 1. Overview of the jet on HMI magnetograms (top row) and AIA $304 \AA$ images (bottom row). The jet including twice eruptions, 'Jet1' and 'Jet2', occurred in the subregion outlined by the dotted boxes. DAVE flows in the first two frames are averaged over 00:00-03:52 UT and 01:37-03:30UT, respectively. The red (green) arrows indicate the direction and the magnitude of the horizontal velocity of positive (negative) polarity elements. P1 denotes a unipolar region of network flux while $\mathrm{n}$ and $\mathrm{p}$ represent an emerging bipole. The field of views (FOVs) are $25^{\prime \prime}$ $\times 25^{\prime \prime}$ for the subregion and $95^{\prime \prime} \times 95^{\prime \prime}$ for other frames. 


\section{Results}

An overview of the jet is displayed by HMI magnetograms and AIA $304 \AA$ chromospheric images in Fig. 1. The red and green arrows indicate the horizontal flow field on the photosphere surface by DAVE method, which show the general supergranular pattern on the quiet-Sun region. There are diverging/convective flows within each supergranule cell (Potts \& Diver 2008) and concentration of network magnetic flux at the edges of each supergranule cell. The dotted boxes indicate the source region of jet eruption with a field-of-view of $25^{\prime \prime} \times 25^{\prime \prime}$ located near the disk center $\left(\mathrm{W} 2{ }^{\circ} \mathrm{N} 15^{\circ}\right)$. In this region, a small-scale bipole (n-p) was emerging from the interior of a supergranule cell from about 01:46 UT, and its negative polarity (n) was swept by photospheric flows to hit the positive, network element (P1) at the cell boundary during 01:46 - 03:11 UT. As a result, P1 was canceled by the collision of $\mathrm{n}$ and a jet with two successive ejections, named as 'Jet1' and 'Jet2', appeared on the chromespheric images in Fig. 1. It is interesting to note that both Jet1 and Jet2 originated where the internetwork bipole interact with the unipolar network element, but had different ejecting directions. The detailed analysis on Jet1 and Jet2 are presented in the following.

Jet1 is shown on EUV images taken by SDO/AIA on disk (panels a-e) and STEREO A/EUVI on limb (panel $f$ ) in Fig. 2. At 02:36 UT, Jet1 started with an EUV brightening occurring at the cancellation site of the negative polarity (n) of the bipole and the positive element (P1). Several minutes later ( panel b), the spire of Jet1 appeared with its footpoint located at the positive polarity (p) of the bipole, and grew toward north in the projected plane. However, it is interesting that Jet1 changed its ejecting direction from northward to northwestward after 02:40 UT. We note that there was a dark, curved EUV filament channel lying to the north of Jet1, which can be clearly seen in panels (a-d) of Fig. 2. The

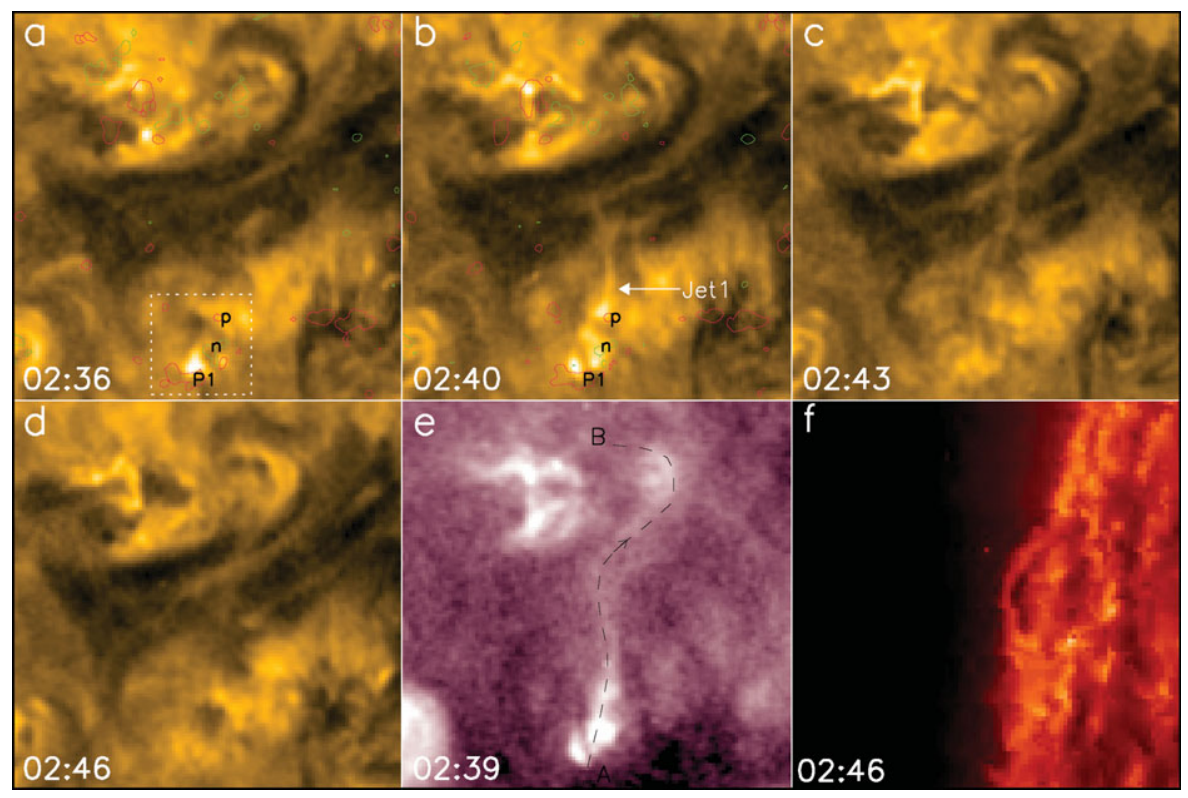

Figure 2. AIA $171 \AA$ images (a-d) showing the on-disk evolution of Jet1 of which the trajectory is clear in $211 \AA$ image (e) and marked by the dash curve A-B. Panel (f) is EUVI $304 \AA$ image showing the on-limb counterpart of Jet1. The red (green) contours in panel (a) and (b) are the levels of HMI magnetic field at $30 \mathrm{G}(-30 \mathrm{G})$. The dotted box and marks (n,p,P1) are the same as in Figure 1. The FOV is $70^{\prime \prime} \times 70^{\prime \prime}$ for panels (a-e) and $140^{\prime \prime} \times 140^{\prime \prime}$ for panel (f). 


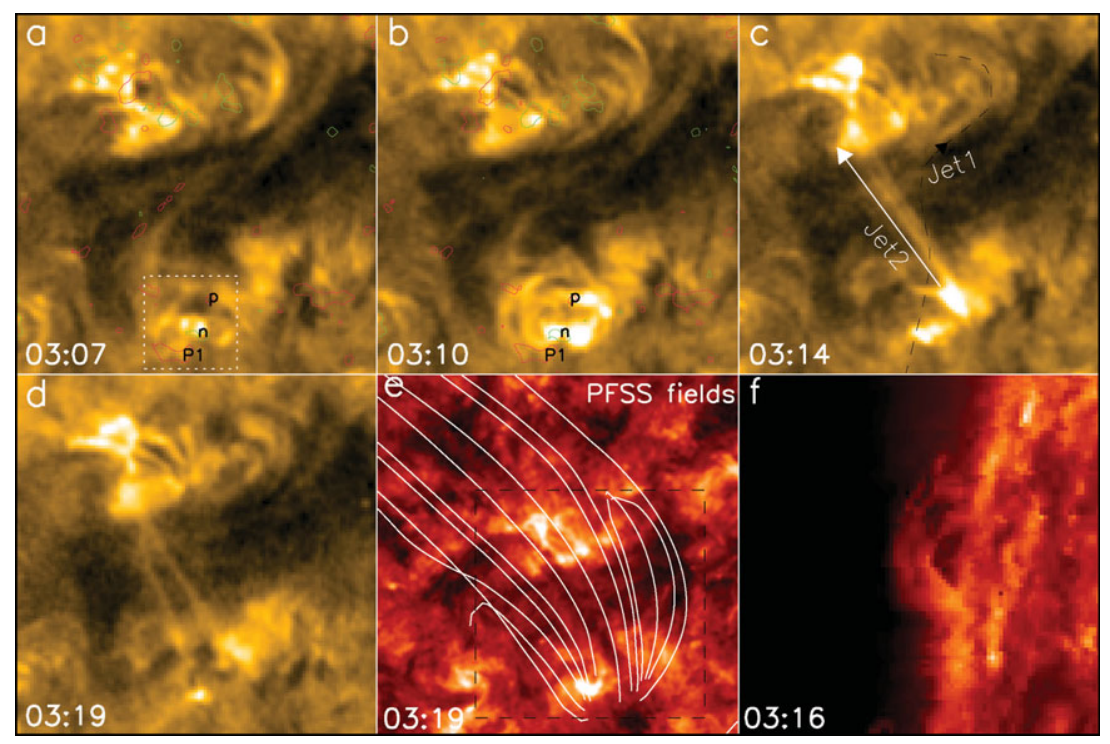

Figure 3. (a-d): AIA $171 \AA$ images showing the on-disk evolution of Jet2. (e): AIA $304 \AA$ image on which PFSS fields is overlaid and the dashed box indicates the FOV in panels (a-d) as same as in Fig. 2(a-d). (f): is EUVI $304 \AA$ image with the same FOV as in Figure 2(f), showing the on-limb counterpart of Jet2. The HMI contours, the dotted box and the marks are the same as in Figure 2.

ejecta turned right to move along the curved filament channel and filled in the northwest portion of the channel ( panel $b$ - $d$ ). It indicated that there should be a direct link between the channel axial fields and the ambient guide fields for Jet1, along which the ejection can be injected into the filament channel (Chae et al. 2000, Wang 1999, Liu et al. 2005, Jiang et al. 2009). The ejection resulted in a distorted trajectory (similar to inverted-S shape) highlighting the link along the curve A-B in the AIA $211 \AA$ image (panel e). Additionally, from the limb view of STEREO-A Jet1 was likely a low arch suggesting that the ejecta was trapped in local magnetic fields.

Jet2 is shown in Figure 3. It also started at the vcinity of the bipole at about 03:05 UT following Jet1. Nevertheless, Jet2 ejected along an entirely different path from Jet1 (see panel $c$ ), although they originated from the same region. Overlying the PFSS fields on a snapshot suggested that Jet2 was consistent with the orientation of the calculated potential field lines that represented high-reching magnetic loops in the quiet-Sun region. Jet2 actually moved along higher magnetic loops on the quite-Sun region than Jet1 by comparison of the near-limb observations (Fig. 2f and Fig. 3f).

\section{Conclusion and discussion}

We study a small-scale jet with two successive plasma ejections on the quiet-Sun region. Our main results are summarized as the following points. (1) The jet was trigged by the magnetic reconnection between the unipolar network field and emerging internetwork bipole at the boundary of a supergranular cell. (2) Its twice ejections had different directions. The first one followed a low, distorted (similar to inverted-S shape) field which guided plasma into the small EUV filament channel nearby. However, the second one shot straight along higher magnetic loops that was parallel with extrapolated potential field lines. 
The fact that the jet moved along two different directions step by step can be interpeted as it followed two kinds of reconnected ambient fields at the supergranular cell boundary where high-reaching magnetic loops coexist with the low, horizontal magnetic canopy (Solanki 1993). The emerging bipole interacted with the high-reaching magnetic loop after with the field of the low magnetic canopy. During this process, the jet followed different reconnected ambient fields from low to high to allow the occurrence of successive ejections along different directions. Additionally, in the quiet-Sun region the low magnetic loops are significantly non-potential while high-reaching loops are quite potential (Woodard \& Chae 1999). It is in agreement with the result that the jet in its first ejection followd the reconnected canopy field that must be non-potential for its distorted shape and linkage with the filament fields, and in the second ejection was parallel with the extarpolated potential fields.

\section{Acknowledgements}

This work is supported by the 973 Program (2011CB811400), by the Natural Science Foundation of China under grant 10973038 and 11173058. The AIA and HMI data used here are courtesy of SDO (NASA) and the AIA/HMI consortia. The EUVI data are courtesy of STEREO and the SECCHI consortium. We thank the AIA, HMI and SECCHI teams for the easy access to calibrated data.

\section{References}

Chae, J., Denker, C., Spirock, T. J., Wang, H., \& Goode, P. R. 2000, Solar Phys., 195, 333

Chen, H.-D., Zhang, J., \& Ma, S.-L. 2012, RAA, 12, 573

Dowdy, J. F., Jr., Rabin, D., \& Moore, R. L. 1986, Solar Phys., 105, 35

Hong, J., Jiang, Y., Zheng, R., et al. 2011, Ap. Lett., 738, L20

Innes, D. E., Genetelli, A., Attie, R., \& Potts, H. E. 2009, A\&A, 495, 319

Jiang, Y. C., Chen, H. D., Li, K. J., Shen, Y. D., \& Yang, L. H. 2007, A\&A, 469, 331

Jiang, Y.-C., Bi, Y., Yang, J.-Y., Zheng, R.-S., \& Wang, J.-X. 2009, RAA, 9, 603

Leighton, R. B., Noyes, R. W., \& Simon, G. W. 1962, ApJ, 135, 474

Lemen, J. R., Title, A. M., Akin, D. J., et al. 2012, Solar Phys., 275, 17

Liu, Y., Kurokawa, H., \& Shibata, K. 2005, Ap. Lett., 631, L93

Martin, S. F. 1988, Solar Phys., 117, 243

Moore, R. L., Cirtain, J. W., Sterling, A. C., \& Falconer, D. A. 2010, ApJ, 720, 757

Parnell, C. E. 2002, MNRAS, 335, 389

Potts, H. E. \& Diver, D. A. 2008, Solar Phys., 248, 263

Schou, J., Borrero, J. M., Norton, A. A., et al. 2010, Solar Phys., 177

Schrijver, C. J. \& De Rosa, M. L. 2003, Solar Phys., 212, 165

Schuck, P. W. 2006, ApJ, 646, 1358

Shen, Y., Liu, Y., Su, J., \& Ibrahim, A. 2011, Ap. Lett., 735, L43

Shen, Y., Liu, Y., Su, J., \& Deng, Y. 2012, ApJ, 745, 164

Shibata, K., Ishido, Y., Acton, L. W., et al. 1992, PASJ, 44, L173

Solanki, S. K. 1993, Space Sci. Rev., 63, 1

Wang, Y.-M. 1999, Ap. Lett., 520, L71

Woodard, M. F. \& Chae, J. 1999, Solar Phys., 184, 239

Wuelser, J.-P., et al. 2004, Proc. SPIE, 5171, 111

Yang, J.-Y., Jiang, Y.-C., Yang, D., et al. 2012, RAA, 12, 300

Yokoyama, T. \& Shibata, K. 1996, PASJ, 48, 353 